\title{
Protective Effects of Danggui Buxue Tang on a Human Umbilical Vein Endothelialcell Damage Induced by Advanced Glycation End Products
}

\author{
Wenyu Qin ${ }^{1,2}$, Bao $\mathrm{He}^{3}$, Yu Bei ${ }^{3}$, Rushang Wang ${ }^{3}$, Lang Feng ${ }^{4}$, Youhua $\mathrm{Xu}^{4}$, Jiaxiao Zhu ${ }^{3}$, \\ Zhaoguang Zheng ${ }^{3}$, Quan Zhu ${ }^{3,4}$, Hong Nie ${ }^{1^{*}}$ \\ ${ }^{1}$ Jinan University College of Pharmacy, Guangzhou, China; ${ }^{2}$ Guangdong Province Key Laboratory of Pharmacodynamic Constitu- \\ ents of TCM and New Drugs Research, Jinan University, Guangzhou, China; ${ }^{3}$ Institute of Conson Co. for Chinese Medicine in Kid- \\ ney Diseases, Consun Pharmaceutical Group, Guangzhou, China; ${ }^{4}$ Faculty of Chinese Medicine, Macau University of Science and \\ Technology, Macau, China. \\ Email: *hongnie1970@163.com
}

Received January $13^{\text {th }}, 2012$; revised February $15^{\text {th }}, 2012$; accepted March $4^{\text {th }}, 2012$

\begin{abstract}
Advanced glycation end products (AGEs) have been regarded as a pivotal inducer in diabetes and kinds of diabetic nephropathy. The present studies explored the effects of Danggui Buxue Tang (DBT) that is a Chinese medicinal decoction on negative charge to Human Umbilical Vein Endothelial Cell (HUVEC) and the related mechanism. Alcian blue staining was established to evaluate the intensity of negative charge on HUVEC. Proteoglycan expressions of AGP and avidin were determined by SDS-PAGE. We observed that DBT can significantly increase negative charge on HUVEC and up-regulated AGP and avidin expressions and ameliorate AGEs-induced HUVEC apoptosis. Therefore, all results showed DBT had prevention effects against the progression of AGEs-induced damage, and this decoction might be promising agent against proteinuria in diabetic nephropathy.
\end{abstract}

Keywords: Danggui Buxue Tang (DBT); AGEs; Negative Charge; Apoptosis; Proteinuria; Diabetic Nephropathy

\section{Introduction}

The World Health Organization estimates that the number of people in the world with diabetes has increased dramatically over recent years and is expected to reach 300 million by the year 2025 [1]. Diabetic complications are recognized as the most common cause of morbidity and mortality in diabetic patients. Diabetic nephropathy (DN), a serious and major complication of diabetes, has become the most common cause of end-stage renal disease (ESRD) in recent years. The first manifestation of diabetic nephropathy in humans is increased urinary albumin excretion, which usually progresses to nephroticrange proteinuria [2]. Increased urinary albumin (proteinuria) is a key component of this disease. Previously, its development led to ESRD with increased morbidity and mortality for diabetic patients versus nondiabetic patients.

Traditional Chinese medicines used to treating nephrosis and diabetic nephropathy for preventing and treating diabetes and its complications [3]. Among thousands of herbal formulae, Danggui Buxue Tang (DBT) is one of

*Corresponding author. the simplest that consists of only two herbs: Radix Astragali (RA, Huangqi) and Radix Angel-icae Sinensis (RAS, Danggui) in a weight ratio of 5:1. The use of DBT was first recorded in "Neiwaishang Bianhuo Lun" by Li Dongyuan in China in 1247 A.D. DBT consists of volatilities, phthalides, organic acids, triterpene saponins and flavonoids [4]. Pharmacological results indicate that DBT possesses the abilities to promote hematopoietic function, stimulate cardiovascular circulation, prevent osteoporosis, increase anti-oxidation activity, and stimulate immune response, increase insulin sensitivity, and decrease albuminuria [4-7]. Moreover, DBT has antiinflammatory properties, and can produce a higher survival rate, less body weight loss, and decrease water intake in diabetic atherosclerosis in GK rats [8]. To date, several interventions have been shown to slow the progression of diabetic nephropathy, Current management strategies for the treatment of diabetic nephropathy have focused on achieving tight glucose and blood pressure control and blockade of the renin angiotensin system. However, none of the currently available modalities can cure or prevent diabetic nephropathy.

Advanced glycation end products (AGEs) in diabetic 
nephropathy have been extensively researched over the last decades and are now firmly established as major players in this disease. Long-term incubation of proteins with glucose leads to the formation of AGEs. The accumulation of AGEs is thought to be a key factor in the initiation and progression of diabetic nephropathy through both receptor dependant and independent interactions [9,10]. And AGEs have been shown to have a wide range of chemical, cellular and tissue effects that potentially could contribute to progressive nephropathy, and modification of proteins leads to changes in charge, solubility and conformation, ultimately resulting in molecular dysfunction [11]. Studies using the remnant kidney model show conflicting data about proteinuria and the use of heparin of the standard drug, and the drug of low weight heparin $[12,13]$. In the mesangioproliferative glomertreatment with heparin can prevent albuminuria and thickening of glomerular basement membranes in puromycin aminonucleoside model $[12,14]$. Heparin is negatively charged, and therefore, it has been suggested that it could replace anionic charges lost by the glomeruli [15].

DBT has been proved clinically effective in reducing proteinuria in chronic kidney disease in China. Despite the medicinal use of DBT dates back thousands of years, the promising evidence from a few studies on the effect of decrease albuminuria, but the mechanisms involved has not been investigated yet. Therefore, in the present study, we wanted to explore the details of DBT at the different dosages and AGEs' effect on negative charges to HUVECs. We also interested in the effects of AGEs on the expression of glycoprotein and HUVECs's apoptosis.

\section{Materials and Methods}

\subsection{Chemical Analysis}

Chromatographic condition was carried out as described by Qi et al. [16]. Alltima C18 $(150 \mathrm{~mm} \times 4.6 \mathrm{~mm}, 5 \mu \mathrm{m})$ column was used, and the mobile phase consisted of water and acetonitrile was changed by concentration gradient of Table 1, the flow rate was $1 \mathrm{~mL} / \mathrm{min}$, the DAD detector was used. Column temperature was constant at 30 centigrade temperature. Standard mixture was purchased from the National Institute for the Control of Pharmaceutical and Biological products (NICPBP). Both Standard mixture and DBT were dissolved with methyl alcohol. The chromatogram was reported within $60 \mathrm{~min}$ (Table 1).

\subsection{Reagents}

Culture reagents were purchased from Invitrogen Technologies (Guangzhou, China). Glycoprotein Molecular
Table 1. Concentration gradient of mobile phase.

\begin{tabular}{ccc}
\hline Time/min & Water (\%) & Acetonitrile (\%) \\
\hline 0 & 1 & 99 \\
10 & 5 & 95 \\
25 & 15 & 85 \\
35 & 25 & 75 \\
60 & 45 & 55 \\
\hline
\end{tabular}

Weight Standards was purchased from Invitrogen Technologies (California, USA), Alcian blue Sigma catalogue no: A5268). Other reagents used were derived from commercial sources.

\subsection{Preparation and Standardization of DBT}

Radix Astragali (RA, Huangqi) and Radix Angelicae Sinensis were bought from Guangzhou Nepstar Chain Drugstore and authenticated by Professor Quan Zhu. A voucher specimen (No. 20090911) was deposited at the Consun pharmaceutical Co.

In preparing DBT, exact amounts of were weighed according to a ratio of 5:1 and then mixed well. The mixture of Radix Angelicae Sinensis and Radix Astragali was immersed in 10 times their total weight of water 30 min, then was boiled for $90 \mathrm{~min}$; Then 10 times their total weight of water was added to the residue and boiled for $90 \mathrm{~min}$ again, and the two extracts were merged and evaporated to dryness under reduced pressure with a rotary evaporator at $50^{\circ} \mathrm{C}$. Finally, the extracts were dried by lyophilization and stored at $-20^{\circ} \mathrm{C}$. Before incubation, the solution was sterile filtered by passing it through a $0.22 \mu \mathrm{m}$ nylon membranes before use.

\subsection{Preparation of Advanced Glycation End Products}

The bovine serum albumin was added into $10 \mathrm{mM} / \mathrm{L}$ phosphate-buffer solution ( $\mathrm{pH} 7.4$, concentration of 5 $\mathrm{g} / \mathrm{L}$ ), incubated with $50 \mathrm{mM} / \mathrm{L}$ D-glucose in $95 \%$ air $/ 5 \%$ $\mathrm{CO}_{2}$ at $37^{\circ} \mathrm{C}$ for 12 weeks. Unincorporated glucose was removed by dialysis overnight against $0.01 \mathrm{M}$ phosphatebuffer solution. AGEs were stored at $-20^{\circ} \mathrm{C}$ until use [17].

\subsection{Culture of Human Umbilical Vein Endothelial Cells}

Human Umbilical Vein Endothelial Cell (HUVEC) line was purchased from the American Type Culture Collection (ATCC, Manassas, VA, USA). Cells were cultured in Dulbecco's Modified Eagle's medium (DMEM, Gibco, USA) supplemented with low glucose, $10 \%$ fetal bovine serum (FBS, Gibco, USA), containing 1\% penicillin-stre- 
ptomycin, at $37^{\circ} \mathrm{C}$ in a $95 \%$ air $/ 5 \% \mathrm{CO}_{2}$ incubator.

\subsection{Alcian Blue Staining}

HUVECs were treated with trypsin and seeded on coverslips precoated with polylysine in 24-well plates at confluence density of $80 \%-90 \%$. HUVECs were starved and incubated with Heparin $(0.05 \mathrm{IU} / \mathrm{ml})$, AGEs (10 $\mu \mathrm{g} / \mathrm{ml})$, DBT/DBT-A/DBT-B $(0.01 \mu \mathrm{g} / \mathrm{ml}, 0.1 \mu \mathrm{g} / \mathrm{ml}, 1$ $\mu \mathrm{g} / \mathrm{ml})+$ AGEs $(10 \mu \mathrm{g} / \mathrm{ml})$ for $1 \mathrm{~h}$. Then the cells were rinsed briefly with phosphate-buffer solution (PBS, $\mathrm{pH}$ 7.4), and fixed with $10 \%$ paraformaldehyde for $10 \mathrm{~min}$ at room temperature. After being fixed, the cells were stained at $37^{\circ} \mathrm{C}$ of water bath with cationic dye Alcian blue solution $(2 \mathrm{mg} / \mathrm{ml}$, with the preparation of $3 \%$ acetic acid) for $30 \mathrm{~min}$. After removal of the stain, washed with tap water for $5 \mathrm{~min}$, and the cells were dehydrated for 5 min with $75 \%, 96 \%, 100 \%$ ethanol and treated with $\mathrm{xy}-$ lene $100 \%$ ethanol for 5 min separately at room temperature. Finally, the cells were treated with xylene and photographed under microscope.

\subsection{Sodium Dodecyl Sulfate Polyacrylamide Gel Electrophoresis (SDS-PAGE)}

Cultured HUVECs were treated with different drugs, and then which were collected in lysis buffer (Beyotime, Shanghai, China) according to manufacture's instructions. Stored in $-20^{\circ} \mathrm{C}$.

The stacking and separating gels used were 5\% and $10 \%$ polyacrylamide, respectively, with an acrylamide: bisacrylerolamide ratio of 29:1. The running buffer was prepared from $25 \mathrm{mM}$ Tris base, $0.2 \mathrm{M}$ glycine, and $0.1 \%$ sodium dodecyl sulfate (SDS) with $\mathrm{pH}$ of 8.3 . Concentrations of bovine serum albumin were determined by the Bradford method. Prior to electrophoresis, the samples were heated in the presence of Sample Loading Buffer (Beyotime, Shanghai, China) at $100^{\circ} \mathrm{C}$ for $5 \mathrm{~min}$ in a boiling water bath. Glycoprotein Molecular Weight Standards was used according to the manufacture's instructions. Glycoprotein samples were loaded into the individual wells, and electrophoresis processed was run at $80 \mathrm{~V}$ for $30 \mathrm{~min}$ in the stacking gels and $120 \mathrm{~V}$ for 60 $\mathrm{min}$ in the separating gels. After separation, the gels were carefully transferred to a petri dish filled with distilled water, The staining solution $(0.1 \% \mathrm{~m} / \mathrm{v}$ Coomassie Brilliant Blue R-250, 50\% v/v methanol, $10 \% \mathrm{v} / \mathrm{v}$ acetic acid) was added into the gel, and then stained overnight at room temperature. Subsequently, the stained gels were removed from the staining solution and distained for $2 \mathrm{~h}$ in $50 \% \mathrm{v} / \mathrm{v}$ methanol and $10 \% \mathrm{v} / \mathrm{v}$ acetic acid, until the background was clear and then washed with distilled water. The gel images were acquired by SensiCapture (Peiqing Science technology Corporation, Shanghai, China) in a WL mode.

\subsection{Cell Apoptosis}

\subsubsection{Fluorescence Detection with Acridine Orange/Ethidium Bromide}

The fluorescence detection with acridine orange/ethidium bromide was used to evaluate the effect of DBT in HUVEC apoptosis. To observe the morphological changes of apoptosis, cells were stained with acridine orange/ ethidium bromide (AO/EB). HUVECs were firstly seeded on the coverslips in 24-well plates at confluence density of $80 \%$. HUVECs were starved and treated with drugs for 48 hours. Then the cells were washed with phosphate-buffer solution (PBS) for one time and incubated with $\mathrm{AO} / \mathrm{EB}$ working solution $(100 \mu \mathrm{g} / \mathrm{ml} \mathrm{AO}$ and 100 $\mu \mathrm{g} / \mathrm{ml}$ ethidium bromide of phosphate-buffer solution). Then cells were observed under a fluorescence microscope. The cytoplasm and nucleus of normal cells were stained with bright green, whereas the apoptotic cells exhibited with jacinth.

\subsubsection{Measurement of Apoptosis by Annexin V/PI Analysis}

HUVECs were firstly seeded on the coverslips in 24-well plates at confluence density of $80 \%$. HUVECs were starved and treated with drugs for 48 hours. After collecting and washing twice with PBS, HUVECs were resuspended in the binding buffer $(200 \mu \mathrm{l})$. FITC-Annexin-V $(2 \mu \mathrm{l})$ was added to the cells followed by addition of $2 \mu \mathrm{l}$ PI according to the protocol of the Annexin V-FITC/PI kit (EMD Biosciences). The samples were then incubated for $15 \mathrm{~min}$ in the dark at room temperature and subjected to flow cytometry evaluation.

\subsection{Statistical Analysis}

Data were expressed as the mean \pm SD. The significance for the difference among groups was analyzed with SPSS16.0 by one-way ANOVAs. Differences were considered to be statistically significance at value of $P<$ 0.05 .

\section{Results}

\subsection{Chemical Analysis}

6 common peaks in HPLC-ELSD fingerprint appeared with retention time could be used as marker peaks for qualitative identification (Figure 1).

\subsection{Assayed by Alcian Blue Staining}

In order to reveal the possible proteinuria-regulatory functions of DBT, water extracts of DBT were applied onto the cultured HUVECs, and the cell negative charge was determined. Alcian blue is a cationic dye which has been used staining of proteoglycan. The staining properties of 


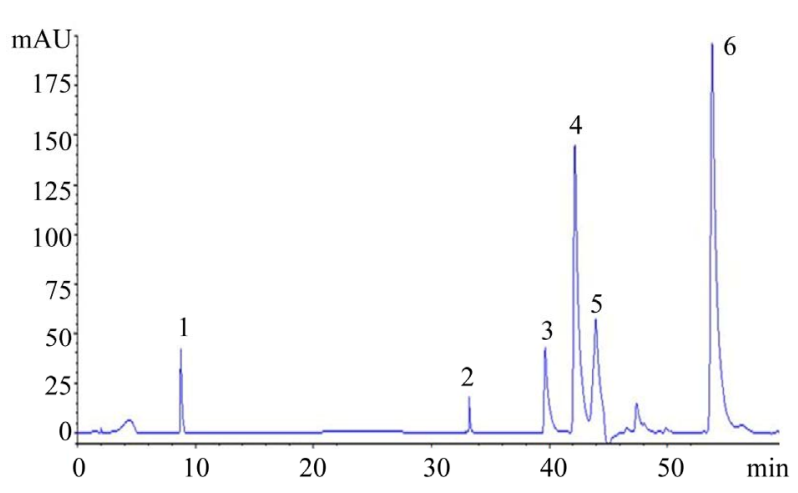

(a)

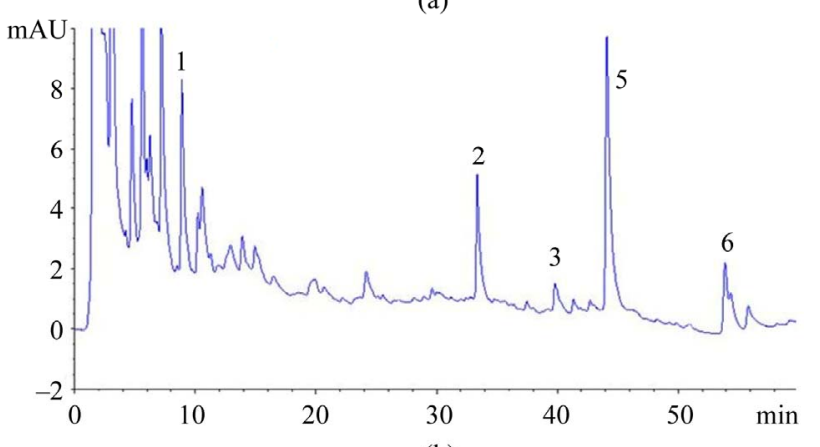

(b)

Figure 1. HPLC of DBT. (a) Six common peaks in HPLCELSD fingerprint appeared. 1: ferulic acid; 2: calycosin-7O-glucoside; 3: ononin-7-O-glucoside; 4: isoflavoues Aglycone; 5: calycosin; 6: ononin; (b) Five common peaks in HPLC-ELSD fingerprint appeared. 1: ferulic acid; 2: calycosin-7-O-glucoside; 3: ononin-7-O-glucoside; 5: calycosin; 6: ononin.

Alcian blue are considered to be associated with the interaction of this cationic dye with negatively charged macromolecules. The data presented here demonstrate that the cells binds Alcian blue, suggesting that these molecules are also negatively charged. As shown in Figure 2, the mean optical density of model group was significantly higher than that of control group $(P<0.01)$. Mean optical density of low, medium, high treatment groups of DBT/DBT-A/DBT-B were sharply increased compared with that of model group $(P<0.01)$. But compared with the heparin group, there were no significantly decreased in DBT, DBT-A and DBT-B treatment groups $(P>0.05)$. The effects of different doses showed a good dose-dependent response. At the dosage of $1 \mu \mathrm{g} / \mathrm{ml}$, DBT showed the sub-maximal effect, which was significantly higher than the other groups.

\subsection{DBT Increased AGP and Avidin Expression in HUVEC}

Glycoproteins were expressed by HUVECs as revealed by SDS-PAGE (Figure 3). Alpha-1-acid glycoprotein (AGP) and avidin are kinds of negatively charge glyco- proteins that cover the surface of cells. Alpha-1-acid glycoprotein (AGP) and avidin expression were significantly decreased in model group compared with control group $(P<0.05)$ and significantly increased in DBT treatment groups compared with the model group $(P<$ $0.05)$. But compared with the heparin group, there were no significantly difference in DBT treatment groups $(P>$ $0.05)$. The effects of different doses showed a good dosedependent response. Notably, there was no significant difference in heparin between control and DBT treatment groups (Figure 4).

\subsection{DBT Protected HUVECs from AGEs-Induced Apoptosis}

To observe the morphological changes of the cells, HUVECs were stained with acridine orange/ethidium bromide solution and observed under a fluorescence microscope. As shown in Figure 5, control group was stained with bright green, and no obvious jacinth-staining was observed; while AGEs $(1 \mathrm{mg} / \mathrm{ml})$ treatment obviously increased the intracellular jacinth staining (Figure 5(b)), indicating more cell apoptosis appeared. As expected, treated with aminoguanidine and DBT were obviously reduced the jacinth staining (Figures 5(c)-(f)). HUVECs were treated with DBT at different concentrations for 48 hours. Apoptotic cells were measured by Annexin V/PI staining. As Figure 6 treated with aminoguanidine and DBT were obviously reduced the apoptosis of HUVECs.

\section{Discussion}

Diabetes is a multisystem disorder and involvement of the kidney is a major cause of hospitalization and infirmity among the diabetic population [18]. The health implications of diabetes is a chronic disorder of metabolism caused by an absolute or relative lack of insulin and its incidence is considered to be about $5 \%$ all over the world. Diabetes is the single leading cause of end stage renal disease in the western world, and is increasing rapidly as its cause in developing countries [2,19]. Diabetes's complications remain poorly understand, which has been the bottle neck in the development of effective therapeutic strategies to the prevention and treatment of diabetes and its complications. Moreover, diabetic nephropathy is the most serious life limited complication of both types 1 and 2 diabetes mellitus, and has been the cause of lot of morbidity and mortality in the diabetic population. It is characterized by increased urinary albumin excretion and loss of renal function. AGEs have been widely studied in its key role in promoting diabetes development [20]. Furthermore, there is increasing evidence that AGEs play an important role in diabetic renal disease. In the present study, we demonstrated that DBT can significantly increase AGEs-induced negative charge to HUVEC, in 
(a)

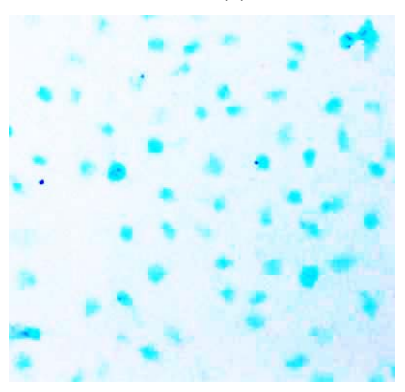

(e)

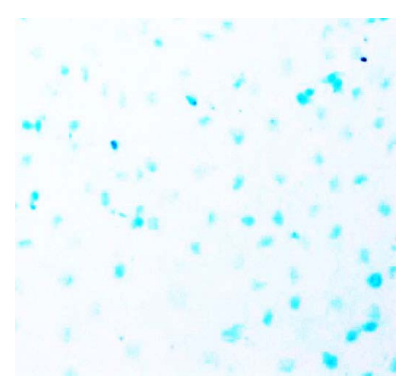

(i)

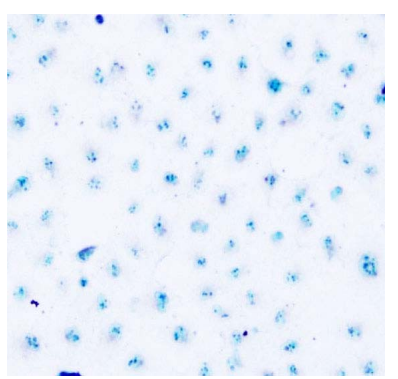

(b)

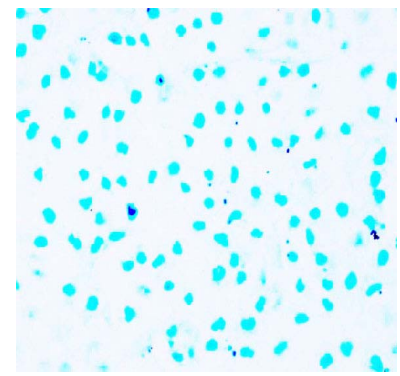

(c)

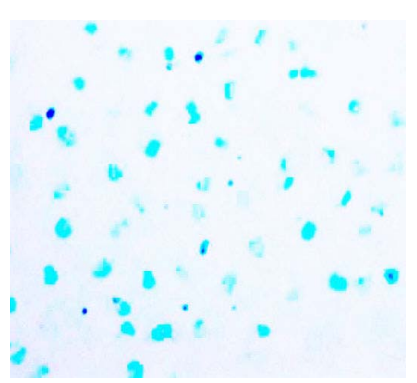

(d)

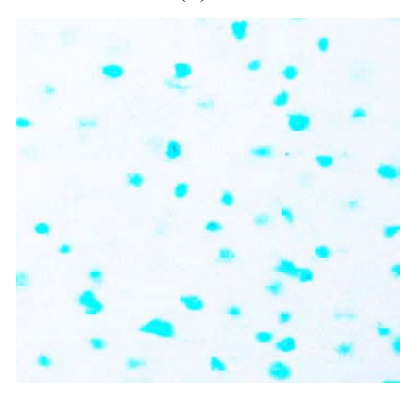

(f)

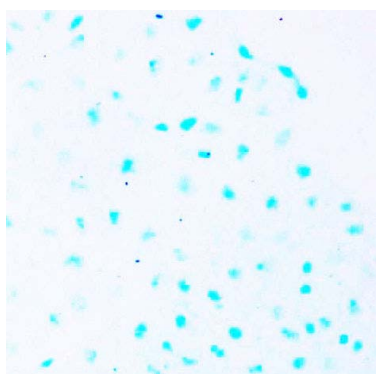

(g)

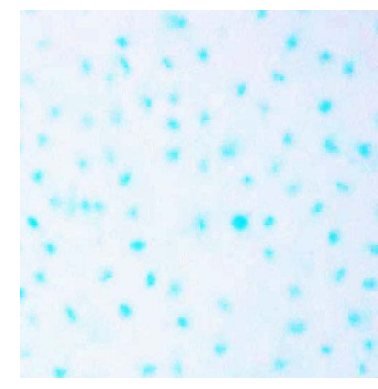

(h)

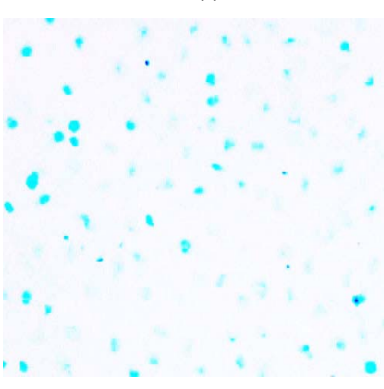

(j)

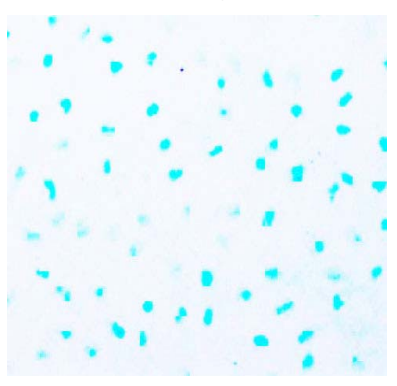

(k)

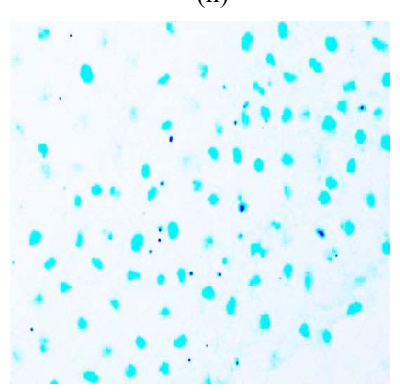

(1)

(A)

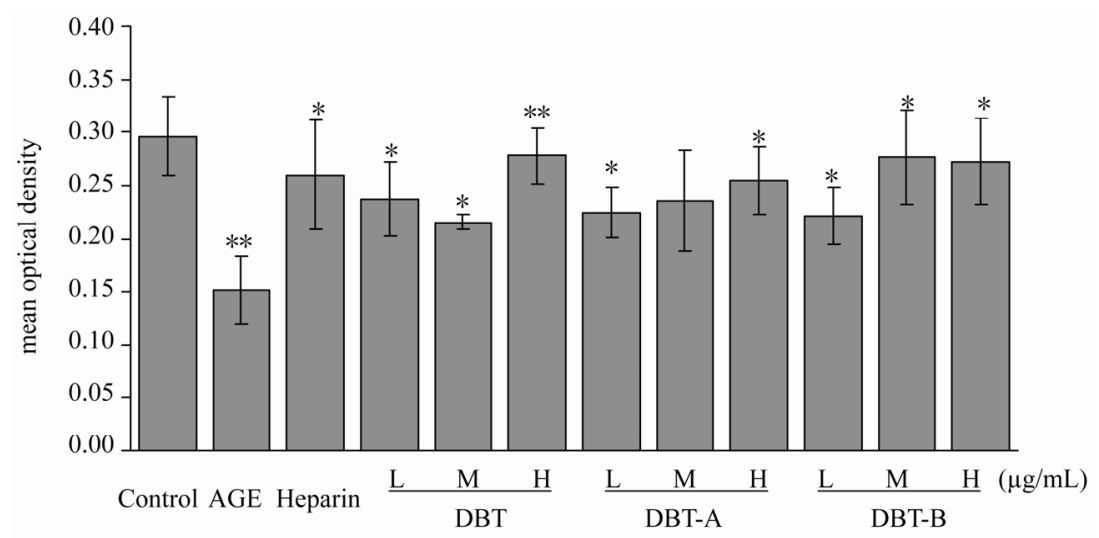

(B)

Figure 2. Alcian blue staining. HUVECs were treated with $10 \mu \mathrm{g} / \mathrm{ml}$ AGEs(Ab), 0.05 IU/ml Heparin + $10 \mu \mathrm{g} / \mathrm{ml}$ AGEs(Ac), $0.01 \mu \mathrm{g} / \mathrm{ml}$ DBT + $10 \mu \mathrm{g} / \mathrm{ml}$ AGEs(Ad), $0.1 \mu \mathrm{g} / \mathrm{ml}$ DBT + $10 \mu \mathrm{g} / \mathrm{ml}$ AGEs(Ae), $1 \mu \mathrm{g} / \mathrm{ml}$ DBT + $10 \mu \mathrm{g} / \mathrm{ml}$ AGEs(Af), $0.01 \mu \mathrm{g} / \mathrm{ml}$ DBT-A + $10 \mu \mathrm{g} / \mathrm{ml}$ AGEs(Ag), $0.1 \mu \mathrm{g} / \mathrm{ml}$ DBT-A + $10 \mu \mathrm{g} / \mathrm{ml}$ AGEs(Ah), $1 \mu \mathrm{g} / \mathrm{ml} \mathrm{DBT-A} \mathrm{+} 10 \mu \mathrm{g} / \mathrm{ml}$ AGEs(Ai), 0.01 $\mu \mathrm{g} / \mathrm{ml}$ DBT-B + $10 \mu \mathrm{g} / \mathrm{ml}$ AGEs(Aj), $0.1 \mu \mathrm{g} / \mathrm{ml}$ DBT-B + $10 \mu \mathrm{g} / \mathrm{ml}$ AGEs(Ak), $1 \mu \mathrm{g} / \mathrm{ml}$ DBT-B + $10 \mu \mathrm{g} / \mathrm{ml}$ AGEs(Al) for $1 \mathrm{~h}$ and staining for $30 \mathrm{~min}$. Negative control (Aa) (Magnification, 200×). DBT increased mean optical density in AGEs-induced HUVECs (B). The staining was quantitated by Image-Pro Plus software and the difference between groups was compared. The date was expressed as means $\pm \mathrm{SD},{ }^{*} p<0.05,{ }^{* *} p<0.01$. The experiment was repeated for at least three times, and representatives were shown. (DBT-A: DBT solution was filtrated with an ultrafiltration membrane of molecular weight 5 kDa, DBT-B: DBT solution was filtrated with an ultrafiltration membrane of molecular weight $10 \mathrm{kDa})$. 


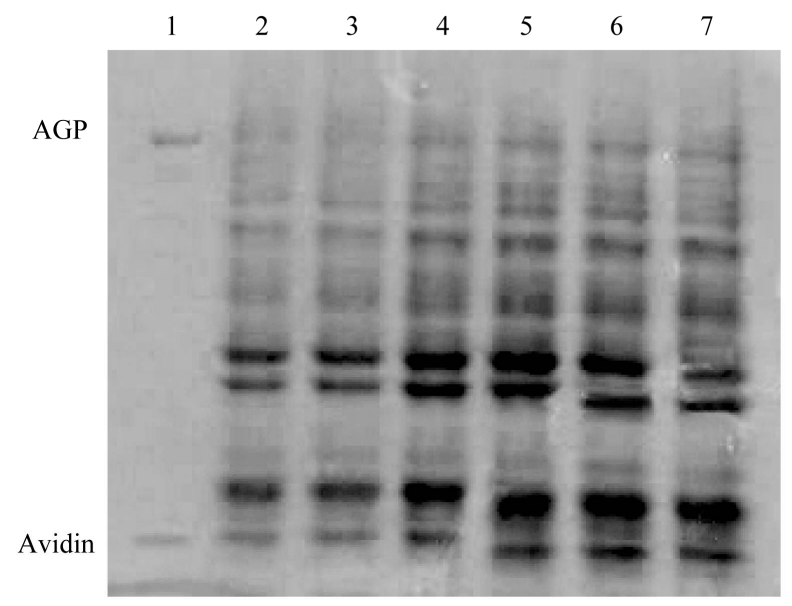

Figure 3. Sodium dodecyl sulfate-polyacrylamide gel electrophoresis results for membrane glycoprotein of HUVECs: lane 1, Glycoprotein; lane 2, Negative control; lane 3, AGEs; lane 4, Heparin; lane 5, DBT low treatment group; lane 6, DBT medium treatment group; lane 7, DBT high treatment group. Coomassie blue stain.

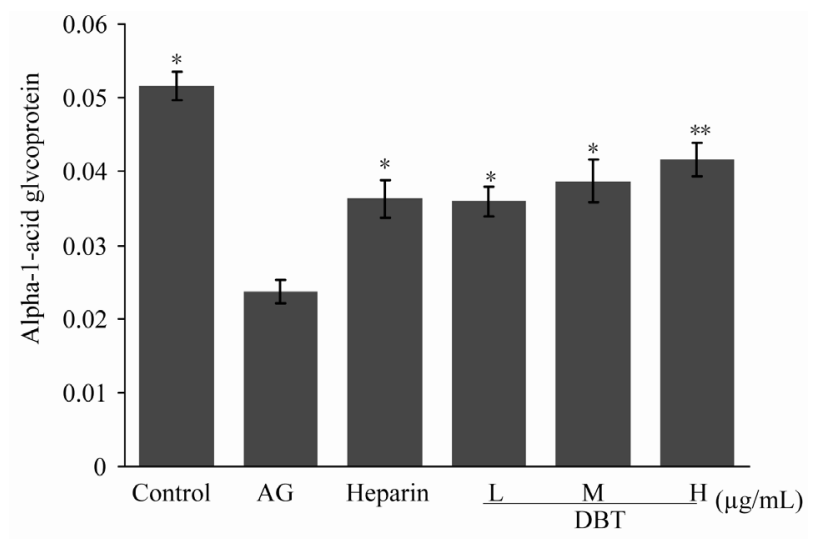

(a)

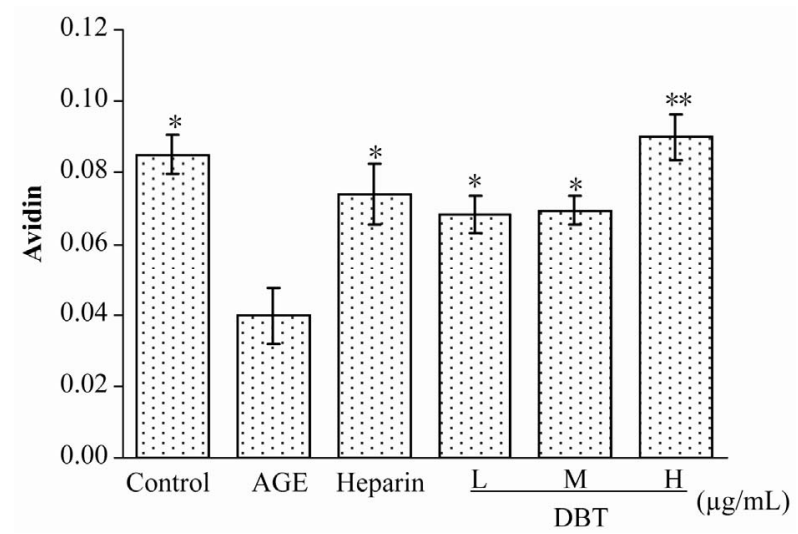

(b)

Figure 4. DBT increased Alpha-1-acid glycoprotein (AGP) and Avidin expression in AGEs-activated HUVECs. The date was expressed as means $\pm \mathrm{SD},{ }^{*} p<0.05,{ }^{* *} p<0.01$. The experiment was repeated for at least three times, and representatives were shown.

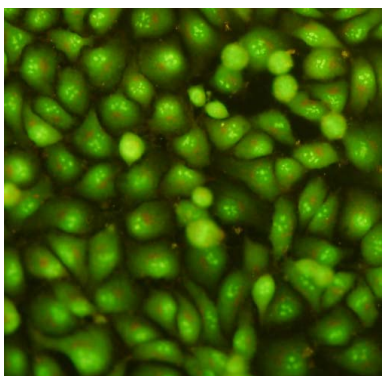

(a)

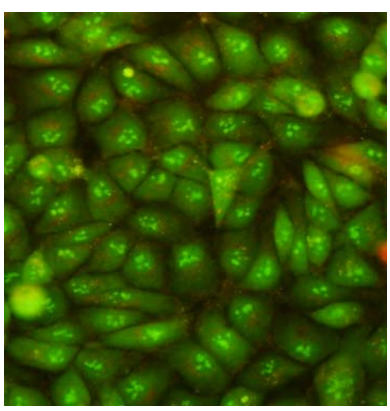

(c)

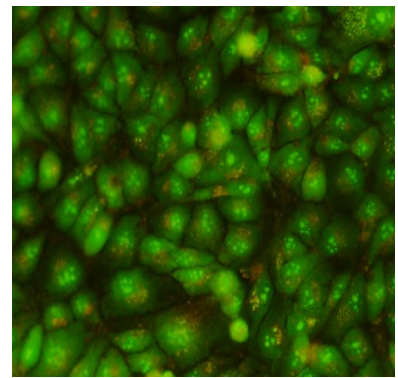

(e)

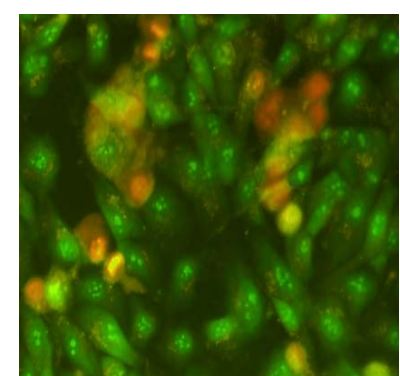

(b)

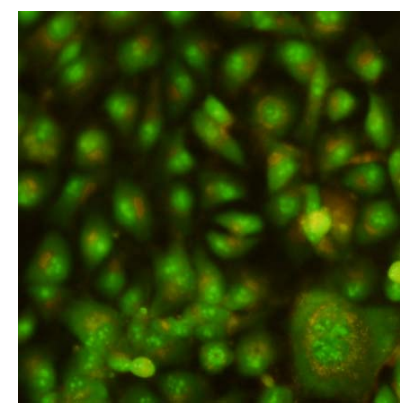

(d)

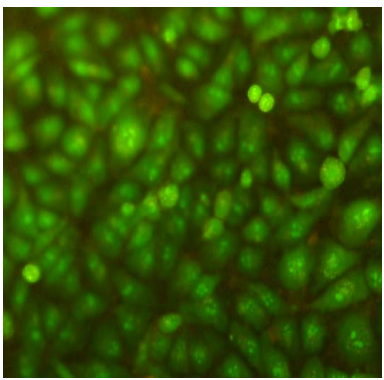

(f)
Figure 5. DBT protected HUVECs from AGEs-induced apoptosis. Negative control (a); HUVECs were incubated with $1 \mathrm{mg} / \mathrm{ml}$ AGEs (b); $100 \mathrm{uM}$ Aminoguanidine + $1 \mathrm{mg} / \mathrm{ml}$ AGEs (c); $0.01 \mu \mathrm{g} / \mathrm{ml}$ DBT + $1 \mathrm{mg} / \mathrm{ml}$ AGEs(d); $0.1 \mu \mathrm{g} / \mathrm{ml}$ DBT $+1 \mathrm{mg} / \mathrm{ml}$ AGEs (e); and $1 \mu \mathrm{g} / \mathrm{ml}$ DBT $+1 \mathrm{mg} / \mathrm{ml}$ AGEs (f) for $48 \mathrm{~h}$ and HUVECs were stained with AO/EB solution. The jacinth staining indicates cell apoptosis status.

which up-regulation of glycoprotein's expression and protected HUVEC from AGEs-induced apoptosis.

The intricate properties of the glomerular barrier have fascinated researchers for decades. The glomerular barrier is by far the most complex biological membrane, with properties that allow for high filtration rates of water, nonrestricted passage of small and middle-sized molecules, and almost total restriction of serum albumin and larger proteins [21]. The glomerular filtration barrier consist of kinds of layers in series podocytes, glomerular basement membrane, fenestrated endothelial cells and endothelial cell surface layer (ESL, often referred to as the glycocalyx) [21]. The glomerular barrier is highly size and charge selective, however, since all components of the glomerular membrane are important for its func- 

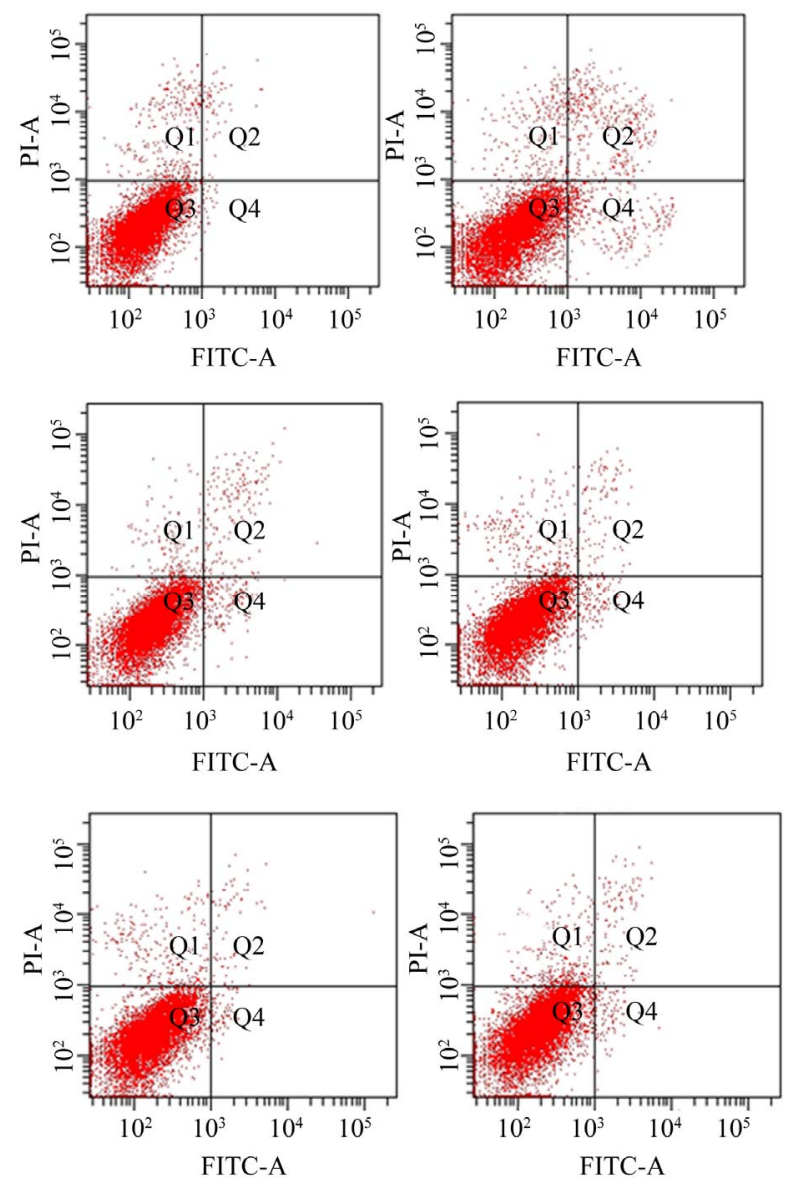

Figure 6. Effects of DBT on the expression of Annexin $V$ on HUVECs. Apoptotic cell death was induced by $1 \mathrm{mg} / \mathrm{ml}$ AGEs. DBT $(0.01 \mu \mathrm{g} / \mathrm{ml}, 0.1 \mu \mathrm{g} / \mathrm{ml}, 1 \mu \mathrm{g} / \mathrm{ml})$ was added to the cultures for $48 \mathrm{~h}$. The cells were stained with Annexin V-fluorescein isothiocyanate (FL-1) and propidiumiodide (PI) (FL-2). Q2 denotes late apoptotic and necrotic cells. Q4 denotes early apoptotic cells (Annexin V positive and PI negative). Q1 + Q2 represent total dead cells. These data demonstrated that AGEs significantly increased cell death (model vs normal), and DBT significantly reduced apoptosis and total cell death (DBT vs model), ${ }^{*} p<0.05,{ }^{* *} p<$ 0.01.

tion, proteinuria will occur regardless of which layer is defect. Not surprisingly, in humans there is a close relationship between renal function, including proteinuria and glomerular filtration rate [19]. Proteinuria is said to be present when the urine contains more than $300 \mathrm{mg}$ protein per day, urine with daily protein excretion ranging from 30 to $300 \mathrm{mg}$ reflects microalbuminuria. Diabetic nephropathy has several stages: microalbuminuria; proteinuria and chronic renal insufficiency with decreased renal filtration and elevated levels of uremic toxins in blood [22]. It is evident that regardless of where the defect is in any of the glomerular layers may result in proteinuria, owing to abnormality in both size selectivity and charge selectivity. Namely, proteinuria with resultant diminution in the physiological electrostatic charge barrier and size barrier.

The importance of proteinuria, in the detection and consequences of glomerular disease has prompted, many studies designed to elucidate the relationship of normal glomerular structure to the normal function of the glomerulus as a barrier to protein filtration, and the specific alterations in structure responsible for the abnormal glomerular permeability to macromolecules observed in disease. With the majority of studies suggesting that net negative charge on a macromolecule reduces filterability and that net positive charge increases filterability. This effect has been attributed to the fixed negative charges associated with all three components of the filtration barrier. Charged molecules also play an important structural role, since their neutralization leads to changes in the size dependence of permeability [23]. Among the risk factors that influence the likelihood of developing renal disease in type 2 diabetes, the onset of proteinuria often heralds a more rapid decline in renal function [24].

Traditional Chinese medicines (TCMs) have been used as medicines, or health supplements, in China over thousands of years. Historically, TCMs are prepared as decoctions by a unique methodology with specific combination of different herbs as a formula. A Chinese medicinal decoction, DBT is a sample combination of two herbs. A number of clinical patients and studies have suggested that these agents may have renoprotective actions against from proteinuria. In the present study we confirmed our observation that DBT is important mediator of AGEs-induced to HUVECs damage.

Recently, there has been a renewed interest in the cell surface coat that is produced by and surrounds the endothelial cells [21]. This layer has two components: the glycocalyx and the endothelial cell coat. The ESL is composed of negatively charged glycoproteins, glycosaminoglycans (GAGs) and membrane-associated and secreted proteoglycans, and can be visualized by using different dyes, for example, Alcian blue is a cationic dye which has been used staining of proteoglycans. Proteoglycans are versatile molecules found in diverse areas of the body and present on the surface of endothelial cells and podocytes and in the mesangial cell matrix as well. The hallmark of a proteoglycan is constituted of a core-protein carrying at least one GAG chain. In diabetic nephropathy, the total amount and charge of GAG levels are modified, potentially contributing to albuminuria. The negative charges in podocytes are thought to contribute to charge selectivity, podocyte stability, and signaling. Podocytes influence endothelial properties by secreting substances such as vascular endothelial growth factor (VEGF) and angiotensin I. As mentioned above, 
the surface anionic charge is essential for maintaining the glomerular structure and function. In the glomerulus, the most described proteoglycans are perlecan [25] and agrin [26,27], both expressed in the GBM together with another proteoglycan. In addition, treatment with puromycin aminonucleoside down regulated proteoglycan expression in glomerular endothelial cells in rats [28]. Alpha-1-acid glycoprotein (AGP) and avidin are kinds of negatively charge glycoproteins that cover the surface of cells. In addition, we treatment with DBT up regulated Alpha-1-acid glycoprotein (AGP) and avidin expression in HUVECs and induced by AGEs.

AGEs accumulates in diabetes and reduction of AGE formation ameliorates the development of diabetic nephropathy [29]. AGEs were believed to be the most important damage factor which effect may be both direct (through AGE receptors) and indirect in the occurrence and development of endothelial dysfunction in diabetic complications. Receptor for advanced glycation end products (RAGE) is an immunoglobulin protein that mediates AGEs induced cell damage process, and is minimally expressed in normal tissue and vasculature and is up-regulated when AGE ligands accumulate. Apoptosis plays an important role in renal development physiology and pathology; studies indicated that endothelial cells apoptosis is of great significance in the progression of diabetic nephropathy, in which it can cause the loss of endothelial cells. The reduction of the endothelium nonadhesive propertly, and the occurence of the vascular dysfunction [30,31]. The published studies have also shown that AGE-RAGE interaction can induce apoptosis and inflammation in diabetic vascular complications, and promote diabetic-associated vasculopathy development [32-35]. Amino guanidine, a nucleophilic compound, not only decreases the formation of AGEs but also inhibits their action. In the present study, it has been demonstrated that administration of the advanced glycation end products inhibitor aminoguanidine ameliorated the AGEsinduced damage; we found that AGEs significantly induced HUVECs apoptosis as compared with control and DBT pretreatment protected the cell from AGEs-induced damage.

In conclusion, we demonstrated that DBT could ameliorate AGEs-induced HUVEC apoptosis. DBT dramatically attenuated AGEs-induced negative charge, and significantly increased alpha-1-acid glycoprotein (AGP) and avidin expression. Furthermore, DBT pre-treatment decreased HUVECs apoptosis. Therefore it is reasonable to conclude that DBT may be a potential therapeutic agent on ameliorating proteinuria of diabetic complications.

\section{Acknowledgements}

This study was supported by the Guangdong Consun
Pharmaceutical Group. We would like to thank Fei Gu, Tingting Duan, Huiquan Chen, Zhiqun Liu, Dan Tang, and Anguo $\mathrm{Wu}$ for kind help during the study.

\section{REFERENCES}

[1] P. Zimmet, K. G. Alberti and J. Shaw, "Global and Societal Implications of the Diabetes Epidemic," Nature, Vol. 414, No. 6865, 2001, pp. 782-787. doi: $10.1038 / 414782 \mathrm{a}$

[2] K. Susztak, A. C. Raff, M. Schiffer and E. P. Bottinger, "Glucose-Induced Reactive Oxygen Species Cause Apoptosis of Podocytes and Podocyte Depletion at the Onset of Diabetic Nephropathy," Diabetes, Vol. 55, No. 1, 2006, pp. 225-233. doi:10.2337/diabetes.55.01.06.db05-0894

[3] L. Y. Adeline, K. C. Sourris, B. E. Harcourt and V. Thallas-Bonke, "Disparate Effects on Renal and Oxidative Parameters Following RAGE Deletion, AGE Accumulation Inhibition, or Dietary AGE Control in Experimental Diabetic Nephropathy," American Journal of PhysiologyRenal Physiology, Vol. 298, 2010, pp. 763-770. doi:10.1152/ajprenal.00591.2009

[4] T. T. X. Dong, K. J. Zhao, Q. T. Gao, Z. N. Ji, T. T. Zhu, J. Li, et al., "Chemical and Biological Assessment of a Chinese Herbal Decoction Containing Radix Astragali and Radix Angelicae Sinensis: Determination of Drug Ratio in Having Optimized Properties," Journal of Agricultural and Food Chemistry, Vol. 54, No. 7, 2006, pp. 2767-2774. doi:10.1021/jf0531631

[5] H. M. Zhang, S. W. Chen, X. F. Deng, X. G. Yang and X. Huang, "Novel Mutations Found in Mitochondrial Diabetes in Chinese Han Population," Diabetes Research and Clinical Practice, Vol. 76, No. 3, 2007, pp. 425-435. doi:10.1016/j.diabres.2006.09.032

[6] Q. T. Gao, J. K. H. Cheung, J. Li, G. K. Y. Chu, R. Duan, A. W. H. Cheung, et al., "A Chinese Herbal Decoction, Danggui Buxue Tang, Prepared from Radix Astragali and Radix Angelicae Sinensis Stimulates the Immune Responses," Planta Medica, Vol. 72, No. 13, 2006, pp. 1227 1231. doi:10.1055/s-2006-947186

[7] Q. T. Gao, J. K. H. Cheung, J. Li, Z. Y. Jiang, G. K. Y. Chu, R. Duan, et al., "A Chinese Herbal Decoction, Danggui Buxue Tang, Activates Extracellular Signal-Regulated Kinase in Cultured T-Lymphocytes," FEBS Letters, Vol. 581, No. 26, 2007, pp. 5087-5093.

doi:10.1016/j.febslet.2007.09.053

[8] D. S. Raj, D. Choudhury, T. C. Welbourne and M. Levi, "Advanced Glycation End Products: A Nephrologist's Perspective," American Journal of Kidney Diseases, Vol. 35, No. 3, 2000, pp. 365-380. doi:10.1016/S0272-6386(00)70189-2

[9] Q. T. Gao, R. C. Y. Choi, A. W. H. Cheung, J. T. T. Zhu, J. Li, G. K. Y. Chu, et al., "Danggui Buxue Tang-A Chinese Herbal Decoction Activates the Phosphorylations of Extracellular Signal-Regulated Kinase and Estrogen Receptor Alpha in Cultured MCF-7 Cells," FEBS Letters, Vol. 581, No. 2, 2007, pp. 233-240. doi:10.1016/j.febslet.2006.12.018 
[10] K. C. Sourris and J. M. Forbes, "Interactions between Advanced Glycation End-Products (AGE) and Their Receptors in the Development and Progression of Diabetic Nephropathy Are These Receptors Valid Therapeutic Targets," Current Drug Targets, Vol. 10, No. 1, 2009, pp. 42-50. doi:10.2174/138945009787122905

[11] J. M. Bohlender, S. Franke, G. Stein and G. Wolf, "Advanced Glycation End Products and the Kidney," American Journal of Physiology-Renal Physiology, Vol. 289, No. 4, 2005, pp. 645-659. doi:10.1152/ajprenal.00398.2004

[12] J. L. Olson, "Role of Heparin as a Protective Agent Following Reduction of Renal Mass," Kidney International, Vol. 25, No. 2, 1984, pp. 376-382. doi: $10.1038 / \mathrm{ki} .1984 .27$

[13] M. L. Purkerson, D. M. Tollefsen and S. Klahr, "N-Desulfated/Acetylated Heparin Ameliorates the Progression of Renal Disease in Rats with Subtotal Renal Ablation," Journal of Clinical Investigation, Vol. 81, No. 1, 1988, pp. 69-74. doi:10.1172/JCI113312

[14] A. M. R. dos Santos, A. V. de Olveirab, C. C. da SilvaLemos, C. A. Mandarim-de-Lacerdac and R. Bregmana, "Low Molecular Weight Heparin in the Treatment of Puromycin-Induced Nephrosis," Pathology Research and Practice, Vol. 202, No. 3, 2006, pp. 157-163. doi:10.1016/j.prp.2005.11.010

[15] L. B. Jaques. "Heparin: An Old Drug with a New Paradigm," Science, Vol. 206, No. 4418, 1979, pp. 528-533. doi:10.1126/science.386509

[16] L. W. Qi, X. D. Wen, J. Cao, et al., "Rapid and Sensitive Screening and Characterization of Phenolic Acids, Phthalides, Saponins and Isoflavonoids in Danggui Buxue Tang by Rapid Resolution Liquid Chromatography/Diode-Array Detection Coupled with Time-of-Flight Mass Spectrometry," Rapid Communications in Mass Spectrometry, Vol. 22, No. 16, 2008, pp. 2493-2509. doi:10.1002/rcm.3638

[17] Y. H. Xu, S. S. Wang, L. Feng, Q. Zhu, P. Xiang and B. He, "Blockade of PKC-Beta Protects HUVEC from Advanced Glycation End Products Induced Inflammation," International Immunopharmacology, Vol. 10, No. 12, 2010, pp. 1552-1559. doi:10.1016/j.intimp.2010.09.006

[18] T. Chawla, D. Sharma and A. Singh, "Role of the Renin Angiotensin System in Diabetic Nephropathy," World Journal of Diabetes, Vol. 1, No. 5, 2010, pp. 141-145. doi:10.4239/wjd.v1.i5.141

[19] R. Nagai, K. Matsumoto, X. Ling, H. Suzuki, T. Araki, and S. Horiuchi, "Glycolaldehyde, a Reactive Intermediate for Advanced Glycation End Products, Plays an Important Role in the Generation of an Active Ligand for the Macrophage Scavenger Receptor," Diabetes, Vol. 49, No. 10, 2000, pp. 1714-1723. doi:10.2337/diabetes.49.10.1714

[20] G. Orasanu and J. Plutzky, "The Continuum of Diabetic Vascular Disease: From Macro- to Micro-," Journal of the American College of Cardiology, Vol. 53, No. 5, 2009, pp. 35-42. doi:10.1016/j.jacc.2008.09.055

[21] B. Haraldsson, J. Nystroem and W. M. Deen, "Properties of the Glomerular Barrier and Mechanisms of Proteinuria," Physiological Reviews, Vol. 88, No. 2, 2008, pp. 451-487. doi:10.1152/physrev.00055.2006

[22] M. V. Shestakova, I. R. Jarek-Martynowa, N. S. Ivanishina, S. S. Kuharenko, M. N. Yadrihinskaya, et al., "Role of Endothelial Dysfunction in the Development of Cardiorenal Syndrome in Patients with Type 1 Diabetes Mellitus," Diabetes Research and Clinical Practice, Vol. 68, 2005, pp. 65-72. doi:10.1016/j.diabres.2005.03.009

[23] J. A. Bertolatus and L. G. Hunsicker, "Glomerular Sieving of Anionic and Neutral Bovine Albumins in Proteinuric Rats," Kidney International, Vol. 28, 1985, pp. 467-476. doi:10.1038/ki.1985.153

[24] K. V. Lemley, K. Blouch, I. Abdullah, D. B. Boothroyd, P. H. Bennett, B. D. Myers, et al., "Glomerular Permselectivity at the Onset of Nephropathy in Type 2 Diabetes Mellitus," Journal of the American Society of Nephrology, Vol. 11, No. 11, 2000, pp. 2095-2105.

[25] R. V. Iozzo, I. R. Cohen, S. Grassel and A. D. Murdoch, "The Biology of Perlecan: The Multifaceted Heparan Sulphate Proteoglycan of Basement Membranes and Pericellular Matrices," Biochemical Journal, Vol. 302, 1994, pp. 625-639.

[26] S. G. Hagen, A. F. Michael and R. J. Butkowski, "Immunochemical and Biochemical Evidence for Distinct Basement Membrane Heparin Sulfate Proteoglycans," Journal of Biological Chemistry, Vol. 268, No. 10, 1993, pp. 7261-7269.

[27] C. J. Raats, B. J. Vanden, M. A. Bakker, B. OppersWalgreen, B. J. Pisa, H. B. Dijkman, et al., "Expression of Agrin, Dystroglycan, Utrophin in Normal Renal Tissue and in Experimental Glomerulopathies," American Journal of Pathology, Vol. 156, 2000, pp. 1749-1765. doi:10.1016/S0002-9440(10)65046-8

[28] G. A. Bjornson, K. Ebefors, M. A. Saleem, P. W. Mathieson, B. Haraldsson and N. J. Sorensson, "Podocyte Proteoglycan Synthesis Is Involved in the Development of Nephrotic Syndrome," American Journal of Physiology-Renal Physiology, Vol. 292, 2006, pp. 722-730. doi:10.1152/ajprenal.00433.2005

[29] J. M. Forbes, M. E. Cooper, M. D. Oldfield and M. C. Thomas, "Role of Advanced Glycation End Products in Diabetic Nephropathy," Journal of the American Society of Nephrology, Vol. 14, Suppl. 3, 2003, pp. 254-258. doi:10.1097/01.ASN.0000077413.41276.17

[30] L. Feng, Y. H. Xu, S. S. Wang, A. Y. Wai, Z. G. Zheng, R. S. Wang, Q. Zhu, et al., "Preventative Effects of 4,4'Diphenylmethane-bis(methyl) Carbamate Isolated from Cortex Mori on Human Umbilical Vein Endothelial Cell Dysfunction Induced by Advanced Glycation End Products," Phytotherapy Research, Vol. 10, No. 3, 2011, pp. 35-69.

[31] P. O. Bonetti, L. O. Lerman and A. Lerman, "Endothelial Dysfunction: A Marker of Atherosclerotic Risk," Arteriosclerosis, Thrombosis, and Vascular Biology, Vol. 23, No. 10, 2003, pp. 168-175. doi:10.1161/01.ATV.0000051384.43104.FC

[32] T. Matsui, S. Yamagishi, M. Takeuchi, S. Ueda, K. Fu- 
kami and S. Okuda, "Nifedipine, a Calcium Channel Blocker, Inhibits Advanced Glycation End Product (AGE)Elicited Mesangial Cell Damage by Suppressing AGE Receptor (RAGE) Expression via Peroxisome Proliferator-Activated Receptor-Gamma Activation," Biochemical and Biophysical Research Communications, Vol. 385, No. 2, 2009, pp. 269-272. doi:10.1016/j.bbrc.2009.05.061

[33] A. Csiszar and Z. Ungvari, "Endothelial Dysfunction and Vascular Inflammation in Type 2 Diabetes: Interaction of AGE/RAGE and TNF-Alpha Signaling," American Journal of Physiology-Heart and Circulatory Physiology, Vol. 295, 2008, pp. 475-482.

doi:10.1152/ajpheart.00644.2008
[34] J. N. Tsoporis, S. Izhar, H. Leong-Poi, J. F. Desjardins, H. J. Huttunen and T. G. Parker, "Interaction with the Receptor for Advanced Glycation End Products (RAGE): A Novel Receptor-Mediated Mechanism for Myocyte Apoptosis Postinfarction," Circulation Research, Vol. 106, No. 1, 2010, pp. 93-101.

doi:10.1161/CIRCRESAHA.109.195834

[35] T. Ishibashi, M. Kawaguchi, K. Sugimoto, H. Uekita, N. Sakamoto and K. Yokoyam, "Advanced Glycation End Product-Mediated Matrix Metallo-Proteinase-9 and Apoptosis via Renin-Angiotensin System in Type 2 Diabetes," Journal of Atherosclerosis and Thrombosis, Vol. 17, 2010, pp. 578-589. doi: $10.5551 /$ jat.3590 